\title{
Treatment acceleration program and the experience of the DREAM program in prevention of mother-to-child transmission of HIV
}

\author{
Leonardo Palombi ${ }^{\mathbf{a}}$, Maria Cristina Marazzi ${ }^{\mathbf{b}}$, Albertus Voetberg ${ }^{\mathrm{c}}$, \\ N. Abdul Magid ${ }^{d}$ and the DREAM Program Prevention of \\ Mother-To-Child Transmission Team
}

\begin{abstract}
Background: The Drug Resource Enhancement against AIDS and Malnutrition (DREAM) program is a large antiretroviral therapy treatment program financed by the Treatment Acceleration Program (TAP) of the World Bank. In addition to provision of antiretroviral treatment to individuals infected with human immunodeficiency virus (HIV) in sub-Saharan Africa, one major aspect of the DREAM program is nutritional supplementation and prevention of mother-to-child transmission (PMTCT) of HIV.
\end{abstract}

Methods: HIV-positive pregnant women enrolled in the DREAM program receive highly active antiretroviral therapy (HAART) free of charge from the 25th week of gestation, irrespective of clinical stage, CD4 count, and viral load. Their infants receive post-exposure prophylaxis. From 2004 to 2006, women enrolled in the DREAM program in Mozambique, Tanzania, and Malawi received water filters and formula for the first 6 months of lactation. In a second cohort starting in 2005 until 2006 in Mozambique, women received HAART for up to 6 months after delivery and were given the option to breastfeed. We conducted a comparative analysis of the two cohorts of HIV-positive pregnant women followed prospectively and evaluated HIV-1 mother-tochild transmission rates, infant morbidity, and mortality in both cohorts.

Results: In the first cohort, 879 live-born children were delivered, with 809 evaluable infants at 1 and 6 months. In the second cohort, 341 infants were delivered and evaluable at 1 month, and 251 infants were evaluable at 6 months. At age 1 month, HIV-1 transmission rates were $4 / 341(1.2 \%)$ among breastfed infants and $7 / 809(0.8 \%)$ among formula-fed infants. At age 6 months, HIV-1 mother-to-child transmission rates were $2 / 251(0.8 \%)$ among breastfed infants of women receiving HAART and 15/809 (1.8\%) among formula-fed infants $\left(\chi^{2}=0.77, P=0.38[\mathrm{NS}]\right)$. The cumulative incidence rate at 6 months of age was $2.7 \%$ for formula-fed infants and $2.2 \%$ for breastfed infants $\left(\chi^{2}=0.27, P=0.60[\mathrm{NS}]\right)$. There was a trend for HIV-1 infection rates to be slightly greater among formula-fed infants, but overall mother-to-child transmission rates in both cohorts were extremely low. Most infants did relatively well on both feeding regimens. Observed $Z$ scores were greater than among the general infant population in the community. $Z$ scores $\leq 2.0$ for weight by age occurred in 92/809 formula-fed infants $(11.4 \%)$ and in $28 / 251$ breastfed infants $(11.1 \%)$. The rates of anemia in the study infant population were also lower than that of the general population. A hemoglobin value $<8 \mathrm{~g} /$ dl was found in 40/809 formula-fed infants (4.9\%) and in $17 / 251$ breastfed infants $(6.8 \%)$ $\left(\chi^{2}=0.92, P=0.33\right)$. The mortality rate at 6 months of age was 27 per 1000 person-years among formula-fed infants and 28.5 per 1000 person-years in breastfed infants - both considerably lower than the rates of 101 per 1000 person-years observed in Mozambique.

From the ${ }^{\mathrm{a}}$ DREAM Program: Piazza S.Egidio 3/a, 00153, Rome, Italy, the ${ }^{\mathrm{b}}$ University Tor Vergata: Via Montpellier 1, 00133 Rome, Italy, Libera Università Maria Ss. Assunta (LUMSA), Assunta Via della Traspontina, 21 - 00193 Roma, the ${ }^{\mathrm{C}}$ World Bank - AIDS Campaign Team for Africa: 1818 H Street, NW MSN \# G6-082A, Washington DC 20433 USA, and the ${ }^{d}$ DPS Maputo province, Machava General Hospital, Maputo, Mozambique.

Correspondence to Leonardo Palombi, DREAM Program, University Tor Vergata, Via Montpellier 1, 00133 Rome, Italy.

Tel: +39-0672596614; fax: +30-0620427263; e-mail: lepalom@tin.it. 
Conclusions: The DREAM HIV-1 PMTCT protocol was safe and efficacious in reducing transmission in infants of 1 and 6 months of age. Results were comparable to those from developed countries. Breastfeeding among HIV-1 infected mothers receiving HAART posed no additional risk of late postnatal HIV-1 transmission to the infant by 6 months of age.

(c) 2007 Lippincott Williams \& Wilkins

AIDS 2007, 21 (suppl 4):S65-S71

\section{Keywords: breastfeeding, DREAM Program, HIV, mother-to-child transmission, treatment acceleration program (TAP)}

\section{Background}

The Regional HIV/AIDS Treatment Acceleration Program (TAP) is a World Bank (International Development Association [IDA]) grant-financed program comprising five grant agreements between the IDA and the governments of Mozambique, Ghana, and Burkina Faso, and the United Nations Economic Commission for Africa (UNECA) and the World Health Organization (WHO) for a combined total amount of US $\$ 60$ million. The project was approved by the Board of Directors on 17 June 2004 and the five Development Grant Agreements became effective on various dates between 22 November 2004 and $25 \mathrm{March}$ 2005 . This project was inspired by an initial hesitance of some governments to provide antiretroviral treatment in the face of many technical, financial, and sustainability issues. The primary goal of the project was to pilot strategies for strengthening each country's capacity to scale up comprehensive human immunodeficiency virus (HIV)/acquired immunodeficiency syndrome (AIDS) programs providing care and treatment in an effective, affordable, and equitable manner. Over a 3-year period, TAP is financing three project components: testing approaches for scaling up service delivery for HIV/AIDS care and treatment; strengthening institutional capacity for HIV/AIDS care and treatment; facilitating information sharing among the TAP countries and technical learning at the regional level. Care and treatment components in the TAP project are provided through partnerships between each government and 'Implementing Partners'. A faith-based organization, the Community of Sant'Egidio is one of three implementing partners in Mozambique, along with Health Action International and Pathfinder. Sant'Egidio is responsible for the Drug Resource Enhancement against AIDS and Malnutrition Programme (DREAM). The basic structure of the DREAM program is described elsewhere [1]. Table 1 shows details of the package supplied by the DREAM program and a brief list of activities undertaken.

\section{Introduction}

In Mozambique today, with TAP support, the DREAM program monitors and provides assistance to more than 15000 HIV-positive people, a significant portion of whom enrolled during the past 2 years. More than $50 \%$ of the cohort are receiving highly active antiretroviral therapy (HAART), and the remaining 7000 enrolled patients are expected to begin treatment in the next 3 years. The experience of recent years has highlighted that it is both feasible and cost-effective to administer HAART in Africa [2]. The program has demonstrated that there are many special circumstances that should be taken into consideration in managing patients from lowincome settings. The additional burden of disease including malnutrition, tuberculosis, anemia, malaria, and parasitosis - aggravates HIV disease itself [3,4] and in many cases appears to accelerate mortality, both in children and in adults [5-7]. It appears that western guidelines for initiation of antiretroviral treatment in HIV-positive patients do not take into account the background morbidity common in African countries, and initiation of treatment at higher strata of CD4 counts and in patients with lower body mass index may be warranted [8]. Facilitating delivery of antiretroviral care continues to be a major priority of DREAM, but several challenges remain.

With the development of treatment protocols for HIVpositive patients in Africa, it became evident that one of the main areas of focus of the DREAM program should include prevention of mother-to-child transmission (PMTCT) of HIV. Despite the proven efficacy of

Table 1. The Drug Resource Enhancement against AIDS and Malnutrition (DREAM) package.

A nationwide public health program that encourages cooperation between faith-based and local non-governmental organizations, the DREAM program provides a full package of care free of charge, including:

- Education and social support ${ }^{\mathrm{a}}$

- Voluntary counselling and testing

- Highly active antiretroviral therapy (HAART) since late February 2002

- Treatment of opportunistic infections, sexually transmitted diseases, and malaria

- Nutritional evaluation and supplementation

- Laboratory diagnostic support including measures of viral load and CD4 cell subsets

- Mother-and-child HIV prevention and care

- Home care

- Information technology and data management

- Operational research

${ }^{\mathrm{a}}$ Training courses are provided locally and internationally for Medical Doctors, technicians, biologists, nurses, social workers, and activists. 
antiretroviral drugs in the interruption of HIV-1 transmission, there remain significant barriers to the implementation of PMTCT [9-11], including very limited access to health centers (in Africa, quite frequently, access is less than $50 \%$ ), high refusal rates for HIV testing, and high rates of drop out from prenatal care. In addition, there remains the problem of development of increased viral resistance mutations with the regimens locally available, particularly with the use of nevirapine-based HAART regimens [12], all of which are a threat to succeeding pregnancies and the prospect of future treatment for mothers and infants [13,14]. There are also the issues of breastfeeding and HIV transmission: breastfeeding, although conferring significant protection from infant mortality, has a significant risk of transmission of HIV. Finally, many PMTCT programs fail to provide continuing treatment and care to mothers. It is difficult to have to tell a woman that she can avoid transmitting the infection to her child, but that little can be done for her own health. Under these circumstances, the refusal rates and no-return rates for those who are tested remain high. Nevertheless, in our experience, when proposals to protect the unborn child are accompanied by an immediate offer of treatment to the mother, outcomes improve significantly.

In order to circumvent these issues, the DREAM program opted for the administration of HAART to all HIV-positive pregnant women irrespective of their virologic, immunologic, and clinical status. The results are very encouraging: we observed significant patient adherence (81\%), extremely low rates of transmission of HIV from mother to child for women who completed the full treatment protocol (1.4-3.8\%), and significant reduction in the presence of mutations associated with antiretroviral resistance $[8,15,16]$. When the DREAM PMTCT program was initiated, in conjunction with antiretrovirals and nutritional supplementation, formula and water filters were distributed for the first 6 months of life. However, it soon became clear that this approach, although very effective [8], was technically difficult and extremely expensive. In practice, 6 months of formula feeding was as costly as 6 months of HAART, presenting an insurmountable stumbling block for scaling up the intervention. Therefore, we conducted a comparative analysis of breast milk characteristics among women treated or not treated with antiretroviral drugs during lactation. Results demonstrated that the risk of detectable breast milk viremia was four times greater in untreated women. Detectable levels of HIV RNA were noted only in a low proportion of women receiving HAART, suggesting a powerful protective impact of treatment $[17,18]$ (Figure 1). Given these results and the difficulties of formula feeding, patients enrolled in our program were subsequently offered the choice of breastfeeding, with continuation of maternal HAART for 6 months after delivery. In order to validate our results, we compared data on the safety and efficacy of formula feeding of infants in the first 6 months of age in preventing late

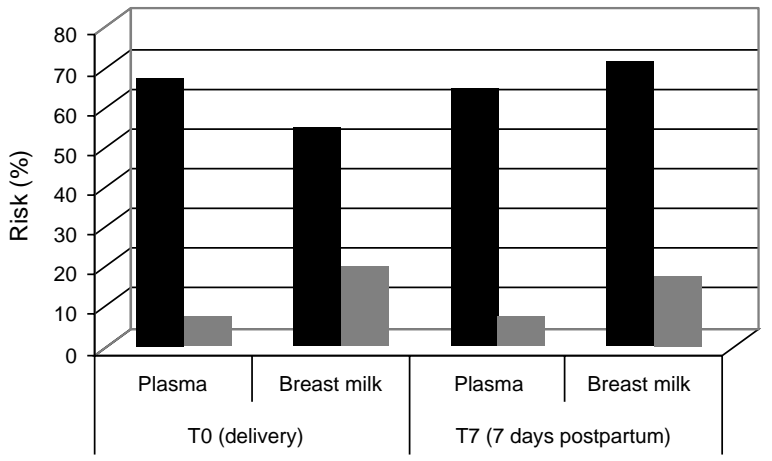

Fig. 1. Risk of detectable HIV-1 levels in breast milk among women not receiving (dark bars) or receiving (lighter bars) HAART after pregnancy. Odds ratios: 4.8 (confidence limits 1.7, 13.6) at delivery; 11.7 (confidence limits $3.9,34.1$ ) at 7 days. Delivery: $\mathrm{OR}=4.8(\mathrm{CL}: 1.7-13.6) ; 1$ Week: $\mathrm{OR}=$ 11.7 (CL: 3.9-34.1). @ Non- HAART Group A; HAART Group B.

postnatal transmission of HIV against the safety and efficacy of exclusive breastfeeding by HIV-positive mothers continuing HAART for 6 months postnatally.

\section{Methods}

A description of the DREAM program structure and laboratory diagnostic methodology for PMTCT has been reported previously $[1,19]$. We compared infant morbidity, mortality, and HIV transmission rate data from two prospective observational cohorts of pregnant women enrolled in the DREAM program over time. The first cohort consisted of women who were given supplementary formula and water filters for use during the first 6 months after delivery. The second cohort consisted of women who were given the option to take HAART during the first 6 months postpartum and to breastfeed exclusively, following implementation of the new DREAM protocol guidelines. Rates of mother-to-child transmission of HIV-1 were compared in the two cohorts at the 1st and 6th months of life, in addition to infant morbidity parameters such as anemia and malnutrition and mortality rates.

Statistical analysis included confidence limits exact test, Student's $t$-test, Levine's $F$ test, $\chi^{2}$, odds ratio and Mante-Haentzel adjustment for odds ratio.

\section{Results}

\section{Cohort 1}

The first cohort consisted of pregnant patients enrolled between January 2004 and December 2006 in Mozambique, Malawi, and Tanzania. In this cohort, 879 
Table 2. Breastfeeding compared with formula feeding and rates of transmission of HIV-1 from mother-to-child at the 1st and 6th months of life.

\begin{tabular}{|c|c|c|c|c|c|c|}
\hline & \multicolumn{3}{|c|}{ Month 1} & \multicolumn{3}{|c|}{ Month 6} \\
\hline & HIV +ve (\%) & HIV -ve (\%) & Total observed & HIV + ve (\%) & HIV -ve (\%) & Total observed \\
\hline Breastfeeding & $4(1.2)$ & 337 (99.4) & 341 & $2(0.8)$ & $249(99.2)$ & 251 \\
\hline Formula & $7(0.8)$ & $802(99.2)$ & 809 & $15(1.8)$ & $794(98.2)$ & 809 \\
\hline
\end{tabular}

live-born children were delivered after 914 pregnancies to women who agreed to participate in the DREAM program and completed the treatment protocol. There were 29 sets of twins, 33 stillborn deliveries, and 31 miscarriages. Women who were approached and refused to participate in the program comprised about $14 \%$ of the original cohort, and an additional $4 \%$ of women withdrew from the program during pregnancy. Of 879 infants, 40 were excluded from the analysis because maternal HAART was delivered for less than 1 month during pregnancy. An additional 30 infants (3.4\%) died during the observation period and were excluded from the analysis because a definitive HIV diagnosis was not available.

\section{Cohort 2}

The second cohort included pregnant patients enrolled from August 2005 to July 2006 in Mozambique. In this cohort, 341 infants were born to women who enrolled in the DREAM programme. Patient refusal to participate in this cohort was $11.1 \%$ of the approached patients; an additional $2.5 \%$ of women withdrew from the program during pregnancy. Follow-up is still under way in this cohort; to date, 251 of the 341 infants with data available in the 1 st month of life have completed 6 months of follow-up. In addition, 7/341 infants (2.1\%) died between the two periods and $21(6.2 \%)$ have been lost to follow-up, including two infants who tested HIV positive in the 1st month of life.

Table 2 shows HIV mother-to-child transmission rates observed at the 1st and 6th months of life in both cohorts. In infants aged 1 month, HIV-1 transmission rates were 4/341 among breastfed infants $(1.2 \%)$ and $7 / 809(0.8 \%)$ among formula-fed infants. In infants aged 6 months, HIV-1 mother-to-child transmission rates were $2 / 251(0.8 \%)$ among breastfed infants of women receiving HAART (for the 251 infants with 6 month follow-up data, nearly $74 \%$ of the cohort) and $15 / 809(1.8 \%)$ among formula-fed infants $\left(\chi^{2}=0.77\right.$, $P=0.38$ [NS]). Of interest, HIV-1 infection rates were greater among formula-fed infants. The plausible explanation for this finding is that many women in the formula arm were probably delivering a mixed feeding regimen consisting partly of breast milk and partly of formula. As seen in Tables 3 and 4, the cumulative incidence rate at 6 months of age was $2.7 \%$ for formula-fed infants and $2.2 \%$ for breastfed infants $\left(\chi^{2}=0.27, P=0.60[\mathrm{NS}]\right)$.
Maternal HAART during gestation combined with either of the feeding regimens after delivery was very potent in reducing the risk of acquisition of HIV. Among formula-fed infants, assuming a $25 \%$ overall rate of mother-to-child transmission of HIV, the reduction in risk of acquisition of HIV at 1 month of age was $96.4 \%$. Assuming a late postnatal transmission rate of $15 \%$, the reduction in risk of acquiring HIV was $88 \%$ at 6 months of age. In the breastfed cohort, using the same assumptions, the reduction in risk of acquiring HIV was $95.2 \%$ at 1 month and $94.7 \%$ at 6 months of age.

Most infants did relatively well on both feeding regimens, and observed $Z$ scores were greater than that of the general infant population in the community. $Z$ scores $\leq 2.0$ for weight by age occurred in $92 / 809$ formula-fed infants $(11.4 \%)$ and in $28 / 251$ breastfed infants (11.1\%). The rates of anemia in the infant population in both groups were also lower than in the general population [20]. A hemoglobin value less than $8 \mathrm{~g} / \mathrm{dl}$ was found in $40 / 809$ formula-fed infants $(4.9 \%)$ and in $17 / 251$ breastfed infants $(6.8 \%) \quad\left(\chi^{2}=0.92, \quad P=0.33 \quad[\mathrm{NS}]\right)$. Infant mortality rates were similar for both cohorts.

Table 3. Incidence of HIV-1 in tested children at ages 1 month and 6 months.

\begin{tabular}{lccc}
\hline & 1 Month & 6 Months & $\begin{array}{c}\text { Cumulative } \\
\text { incidence rate }\end{array}$ \\
\hline Formula-fed infants & & \\
HIV+ & $7(0.9)$ & $15(1.8)$ & $22(2.7)$ \\
HIV- & $802(99.1)$ & $794(98.2)$ & $787(97.3)$ \\
Total & $809(100)$ & $809(100)$ & $809(100)$ \\
Breastfed infants & & \\
HIV+ & $4(1.2)$ & $2(0.8)$ & $6(2.2)$ \\
HIV- & $337(99.1)$ & $249(98.2)$ & $266(97.8)^{\mathrm{a}}$ \\
Total & $341(100)$ & $251(100)$ & $266(100)^{\mathrm{a}}$ \\
\hline
\end{tabular}

Values are number (\%).

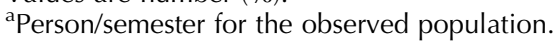

Table 4. Incidence of mother-to-child transmission of HIV-1 over time.

Incidence (95\% confidence limit)

\begin{tabular}{llc}
\cline { 2 - 3 } Time & Formula-fed & Breastfed \\
\hline 1 Month & $0.9(0.3,1.8)$ & $1.2(0.3,3.0)$ \\
TMonths & $1.8(1.0,3.0)$ & $1.8(0.1,2.8)$ \\
Total & $2.7(1.7,4.1)$ & $2.0(0.6,3.8)$ \\
\hline
\end{tabular}


The mortality rate at 6 months of age was 27 per 1000 person-years among the formula-fed infants and 28.5 per 1000 person-years among breastfed infants - notably lower than the rate of 100 per 1000 person-years observed in Mozambique [21].

\section{Discussion}

Delivery of a comprehensive approach to care in the DREAM program has proved very successful, particularly within the context of HIV PMTCT. The retention rates of $80 \%$ or more among our cohorts demonstrate that provision of HAART treatment to pregnant women, along with nutritional supplementation, is a powerful way to enhance adherence to the program. Our data clearly demonstrate that decreasing the rates of transmission of HIV from mother to child in Africa to those reported by high-income countries [22] is feasible, efficacious, safe, and highly achievable even in the poorest of settings. Prenatal administration of HAART, starting as early as 25 weeks of gestation in our program, was highly tolerable and was safe for mothers, as reported previously $[8,19]$. Nevertheless, a post-delivery PMTCT strategy remains crucial for further prevention of late postnatal transmission. Even if the impact of pre- and perinatal interventions is high, the lack of a post-delivery strategy will nullify or significantly reduce the sustainability of results [23-27]. The rate of postnatal transmission of HIV-1 seems to be greatest in the first 6 months of life, although transmission may also occur after this time $[28,29]$. Although we achieved a significant reduction in mother-to-child transmission of HIV and a very low rate of adverse events by providing infant feeding formula, the implementation of this strategy is very difficult because of cost and reliability (the likelihood that women will mix breastfeeding and formula). In our cohort, the use of portable water filters reduced the incidence of gastrointestinal diseases in infants so that excess mortality was not observed (data not shown). Other studies from sub-Saharan Africa demonstrate benefits in reduction of gastroenteritis with breastfeeding alone (without maternal HAART) that apparently outweigh the risks of HIV transmission [30,31].

Our data indicate that the provision of HAART to mothers during the first 6 months of breastfeeding is feasible and highly successful in reducing late postnatal mother-to-child transmission of HIV. This strategy was actually slightly more effective in reducing HIV transmission than formula itself, most probably because women provided mixed feeding when formula was available. Among the breastfed infants, less than $1 \%$ of transmissions occurred beyond 6 months of age, in comparison with an additional $2 \%$ at 6 months in the formula-fed population. Cumulative HIV-1 transmission rates of $2.7 \%$ and $2.2 \%$ at 6 months of age among formula-fed or breastfed infants are extraordinarily low and unusual in the general African setting, and we have demonstrated that this is achievable.

Our population did well with both feeding strategies, with low rates of infant malnutrition as demonstrated by lower rates of $Z$ scores $\leq 2$ than were observed in the general population. There were also lower rates of anemia, with only $5 \%$ of infants in the formula arm and $7 \%$ in the breastfed arm having hemoglobin values less than $8 \mathrm{~g} / \mathrm{dl}$. Anemia is a significant problem in malariaprone Mozambique, with much greater rates observed in the general pediatric population [20]. The fact that mortality rates in both cohorts were only $25-33 \%$ of the reported rates for the country demonstrate the success of the program in improving overall health care and health outcomes.

There are limitations to our study, given the temporal difference between the two cohorts. Nevertheless, even if perinatal transmission rates were declining for reasons unrelated to our intervention, our data indicate that breastfeeding in association with maternal HAART appears to be superior to formula feeding. Another difference is that the first cohort included patients recruited from Malawi and Tanzania, whereas the second cohort was exclusively from Mozambique. Although there could be potential differences in viral subtypes among different regions, the majority of patients in the first cohort were from Mozambique. The third limitation is the smaller number of patients in the second cohort than in the first, and the fact that not all 341 patients had reached the second time-point. Nevertheless, patients continue to be enrolled in the DREAM program, and provision of HAART to mothers during gestation is also continuing. In the future, data from an extremely large group of patients will be available for analysis. However, given the extraordinary findings of extremely low transmission rates among breastfeeding women receiving HAART, and the fact that there are data from a sizeable number of patients, we have chosen to report available results.

In summary, at sites established in Mozambique, Malawi, and Tanzania, the significant success of the DREAM initiative in the overall reduction of HIV-1 transmission rates to levels very similar to those reported in highincome countries reflects the effectiveness of the comprehensive approach delivered by the DREAM and TAP programs. In our experience, four cornerstones are needed to enable the transition from an emergency phase to developing an integrated phase in the struggle against AIDS in Africa. First, there is the need for a system to monitor adherence and the patient's immunologic and virologic status. Secondly, there is great need for implementation of a health education system with involvement of the patient in the treatment process, and availability of formative and review courses for personnel. 
Thirdly, there must be widespread use of antiretroviral treatment for the prevention of HIV, with special attention paid to vertical transmission and protection of HIV-negative partners in serodiscordant couples. Lastly, there is an overwhelming need for a new generation of health centers equipped with the necessary human resources, communication, and coordination technologies, with the ability to mobilize large numbers of patients. This new structure of delivery of care offers a comprehensive system that is explicitly designed for the African environment and is capable of offering more suitable treatment to patients. This is a system based on equity and sustainability that strives for prevention and treatment of disease.

\section{Acknowledgements}

The following are the members of the Dream Program PMTCT Team: Michelangelo Bartolo (S. Giovanni Hospital, Informatic Unit, Rome, Italy); Piero Bestagini, Gabriella Bortolot, Paola Germano, Massimo Magnano San Lio, Graziella Tintisona (Community of Sant'Egidio, Rome, Italy); Ersilia Buonomo, Anna Maria Doro-Altan, Giuseppe Liotta, Sandro Mancinelli, Paola Scarcella, (University Tor Vergata, Rome, Italy); Giovanni Guidotti (National Institute of Health, Rome, Italy); Karin NielsenSaines (University of California Los Angeles, USA).

Disclaimer: The production of this special Supplement was supported by the World Bank, the Joint United Nations Programme on HIVIAIDS and the World Health Organization. The findings, interpretations and conclusions presented in this paper do not necessarily reflect the views of these institutions or their constituent agencies or governments.

\section{References}

1. Marazzi MC, Guidotti G, Liotta G, Palombi L. DREAM: an integrated faith-based initiative to treat HIVIAIDS in Mozambique, case study. Perspectives and practice in antiretroviral treatment. Community of Sant' Egidio and Geneva: World Health Organization; 2005. Available at: http://www.who.int/hiv/pub/ casestudies/mozambiquedream.pdf.

2. Goldie SJ, Yazdanpanah Y, Losina E, Weinstein MC, Anglaret X, Walensky RP, et al. Cost-effectiveness of HIV treatment in resource-poor settings - the case of Cote d'Ivoire. N Engl / Med 2006; 355:1141-1153.

3. Walker AS, Mulenga V, Sinyinza F, Lishimpi K, Nunn A, Chintu C, Gibb DM, the CHAP Trial Team. Determinants of survival without antiretroviral therapy after infancy in HIV-1-infected Zambian children in the CHAP Trial. I Acquir Immune Defic Syndr 2006; 42:637-645.

4. Bronzan RN, Taylor TE, Mwenechanya J, Tembo M, Kayira K, Bwanaisa $L$, et al. Bacteremia in Malawian children with severe malaria: prevalence, etiology, HIV coinfection, and outcome. I Infect Dis 2007; 195:895-904.

5. Minga A, Danel C, Abo Y, Dohoun L, Bonard D, Coulibaly A, et al., the ANRS 1220 Study Group. Progression to WHO criteria for antiretroviral therapy in a 7-year cohort of adult HIV-1 seroconverters in Abidjan, Cote d'Ivoire. Bull World Health Organ 2007; 85:116-123.
6. Braitstein P, Brinkhof MW, Dabis F, Schechter M, Boulle A, Miotti $\mathrm{P}$, et al., the Antiretroviral Therapy in Lower Income Countries (ART-LINC) Collaboration andART Cohort Collaboration (ART-CC) groups. Mortality of HIV-1-infected patients in the first year of antiretroviral therapy: comparison between low-income and high-income countries. Lancet 2006; 367: 817-824.

7. Newell ML, Coovadia H, Cortina-Borja M, Rollins N, Gaillard P, Dabis $F$. Mortality of infected and uninfected infants born to HIV-infected mothers in Africa: a pooled analysis. Lancet 2004; 364:1236-1243.

8. Palombi L. Oral Presentation, Meeting on "Sustaining Treatment Costs: Who Will Pay?" Co-hosted by WHO, UNAIDS, World Bank. Washington, November 27, 2006.

9. Temmerman M, Quaghebeur A, Mwanyumba F, Mandaliya K. Mother-to-child HIV transmission in resource poor settings: how to improve coverage? AIDS 2003; 17:1239-1242.

10. Stringer EM, Sinkala M, Stringer JS, Mzyece E, Makuka I, Goldenberg RL, et al. Prevention of mother-to-child transmission of HIV in Africa: successes and challenges in scaling-up a nevirapine-based program in Lusaka, Zambia. AIDS 2003; 17:1377-1382.

11. Marazzi MC, Germano P, Liotta G, Guidotti G, Louriero S, Gomes AD, et al. Implementation of antiretroviral triple therapy to prevent mother-to-child transmission: a public health approach in resource-limited settings. Eur J Pediatr 2007; [Epub ahead of print Jan 18].

12. Flys TS, Chen S, Jones DC, Hoover DR, Church JD, Fiscus SA, et al. Quantitative analysis of HIV-1 variants with the K103N resistance mutation after single-dose nevirapine in women with HIV-1 subtypes A, C, and D. I Acquir Immune Defic Syndr 2006; 42:610-613.

13. Lockman S, Shapiro RL, Smeaton LM, Wester C, Thior I, Stevens $L$, et al. Response to antiretroviral therapy after a single, peripartum dose of nevirapine. N Engl / Med 2007; 356:135-147.

14. Martinson NA, Ekouevi DK, Dabis F, Morris L, Lupodwana P, Tonwe-Gold $\mathrm{B}$, et al. Transmission rates in consecutive pregnancies exposed to single-dose nevirapine in Soweto, South Africa and Abidjan, Cote d'Ivoire. I Acquir Immune Defic Syndr 2007; [Epub ahead of print Apr 12].

15. Marazzi MC, Bartolo M, Emberti Gialloreti L, Germano P, Guidotti G, Liotta G, et al. Improving adherence to highly active anti-retroviral therapy in Africa: the DREAM programme in Mozambique. Health Educ Res 2006; 21:34-42.

16. Bellocci MC, Forbici F, Palombi L, Gori C, Coelho E, Svicher C, et al. Subtype analysis and mutations to antiviral drugs in HIV-1 infected patients from Mozambique before initiation of antiretroviral therapy: results from the DREAM Programme. / Med Virol 2005; 76:452-458.

17. Giuliano M, Guidotti G, Andreotti M, Pirillo MF, Villani P, Liotta $G$, et al. Triple antiretroviral prophylaxis administered during pregnancy and after delivery significantly reduces breast milk viral load (a study within the Drug Resource Enhancement Against AIDS and Malnutrition Program). I Acquir Immune Defic Syndr 2007; 44:286-291.

18. Giuliano M, Guidotti G, Andreotti M, Liotta G, Cusato M, Marazzi MC, et al. Triple therapy during pregnancy and after delivery significantly reduces breastmilk viral load: a substudy of the DREAM program [abstract No. 727]. In: Abstracts of the 13th Conference on Retrovirus and Opportunistic Infections (CROI); 5-9 February 2006; Denver. p. 313

19. Marazzi MC, Germano P, Liotta G, Guidotti G, Loureiro S, Da Cruz Gomes A, Valls Blazquez MC, Narciso P, Perno CF, Mancinelli S, Palombi, et al. Safety of nevirapine-containing ARV triple therapy regimens to prevent vertical transmission in an African cohort of HIV-1 infected pregnant women. HIV Medicine 2006; 7:338-344.

20. Dorenbaum A, Cunningham CK, Gelber RD, Culnane $M$ Mofenson L, Britto $P$, et al. Two-dose intrapartum/newborn nevirapine and standard antiretroviral therapy to reduce perinatal HIV-1 transmission: a randomized trial. JAMA 2002; 288:189-198.

21. Cartmell E, Natalal $H$, François I, Ferreira MH, Grahnquist L. Nutritional and clinical status of children admitted to the malnutrition ward, Maputo central hospital: a comparison of data from 2001 and 1983. I Trop Pediatr 2005 Apr; 51(2):1021025. Epub 2005 Jan 26. 
22. The Petra Study Team. Efficacy of three short-course regimens of zidovudine and lamivudine in preventing early and late transmission of HIV-1 from mother to child in Tanzania, South Africa and Uganda (Petra study): a randomised double-blind, placebo-controlled trial. Lancet 2002; 359:1178-1196.

23. Nolan ML, Greenberg AL, Fowler MG. A review of clinical trial to prevent mother-to-child transmission in Africa and inform rational intervention strategies. AIDS 2002; 16:1991-1999.

24. Jackson JB, Musoke P, Fleming T, Guay LA, Bagenda D, Allen M, et al. Intrapartum and neonatal single-dose nevirapine compared with zidovudine for prevention of mother-to-child transmission of HIV-1 in Kampala, Uganda: 18-month follow-up of the HIVNET 012 randomised trial. Lancet 2003; 362:859-868.

25. Coovadia HM. Prevention and treatment of perinatal HIV-1 infection in the developing world. Curr Opin Infect Dis 2000; 13:247-251.

26. Lallemant M, Jourdain G, Le Coeur S, Mary JY, Ngo-GiangHuong N, Koetsawang S, et al., the Perinatal HIV Prevention Trial (Thailand) Investigators. Single-dose perinatal nevirapine plus standard zidovudine to prevent mother-to-child transmission of HIV-1 in Thailand. N Eng/ J Med 2004; 351:217-228.
27. Nduati R, John G, Mbori-Ngacha D, Richardson B, Overbaugh J, Mwatha $\mathrm{A}$, et al. Effect of breastfeeding and formula feeding on transmission of HIV-1. JAMA 2000; 283:1167-1174.

28. Miotti PG, Taha TET, Kumwenda NI, Broadhead R, Mtimavalye LA, Van der Hoeven $L$, et al. HIV transmission from breastfeeding: a study in Malawi. JAMA 1999; 282:744-749.

29. Coovadia HM, Rollins NC, Bland RM, Little K, Coutsoudis A, Bennish ML, Newell ML. Mother-to-child transmission of HIV1 infection during exclusive breastfeeding in the first 6 months of life: an intervention cohort study. Lancet 2007; 369:11071116.

30. Taha TE, Kumwenda NI, Hoover DR, Kafulafula G, Fiscus SA, Nkhoma C, et al. The impact of breastfeeding on the health of HIV-positive mothers and their children in sub-Saharan Africa. Bull World Health Organ 2006; 84:546-554.

31. Cartmell E, Natalal H, Francois I, Ferreira MH, Grahnquist L. Nutritional and clinical status of children admitted to the malnutrition ward, Maputo Central Hospital: a comparison of data from 2001 and 1983. J Trop Pediatr 2005; 51:102-105. [Epub 2005 ahead of print Jan 26]. 